\title{
Qualidade de vida em pacientes diabéticos: revisão de literatura
}

\author{
Quality of life in diabetic patients: literature review \\ Calidad de vida en pacientes diabéticos: revisión de literatura \\ Luana Martins Araújo Ribeiro*, Tatiana Martins Araújo Ribeiro1, Ingrid Cristiane Pereira Gomes¹.
}

\begin{abstract}
RESUMO
Objetivo: Avaliar a qualidade de vida em pacientes diabéticos. Métodos: Trata-se de uma revisão integrativa cujo tema foi pesquisado na base de dados do PubMed e Scielo, com descritores "Quality of life AND Diabetes Mellitus" e "Qualidade de vida AND Diabetes Mellitus", respectivamente, sendo incluídos apenas artigos dos últimos três anos. Resultados: Os artigos desenvolvidos através de questionários evidenciaram que a qualidade de vida em diabéticos é menor que em pacientes não diabéticos, devido, principalmente, à mudança do estilo de vida que a doença gera. Tal fato está relacionado também às complicações presentes na ausência ou inadequação do tratamento, assim como à própria terapia farmacológica, às alterações de estilo de vida que são impostas e à ansiedade presente em grande parte desses pacientes. Assim, tais indivíduos têm que lidar com complicações físicas e mentais que a doença proporciona, causando prejuízo no modo de viver. Considerações Finais: Pacientes diabéticos possuem menor qualidade de vida, tanto no âmbito físico como no emocional, quando comparados com indivíduos que não possuem a doença.
\end{abstract}

Palavras-chave: Qualidade de vida, Diabetes mellitus, Complicações.

\begin{abstract}
Objective: To evaluate the quality of life in diabetic patients. Methods: This is an integrative review whose theme was researched in the database of PubMed and Scielo, with the descriptors "Quality of life AND Diabetes Mellitus", only articles from the last three years were included. Results: The articles developed through questionnaires showed that the quality of life in diabetics is lower than in non-diabetic patients, mainly due to the change in lifestyle that the disease generates. This fact is also related to the complications present in the incorrect treatment of the disease, as well as to the pharmacological therapy itself, to the lifestyle changes that are imposed, and to the anxiety present in most of these patients. Thus, such individuals must deal with physical and mental complications that the disease determines, causing damage to the way of life. Final Considerations: Diabetic patients have lower quality of life, both physically and emotionally, when compared to individuals who do not have the disease.
\end{abstract}

Keywords: Quality of life, Diabetes mellitus, Complications.

\section{RESUMEN}

Objetivo: evaluar la calidad de vida en pacientes diabéticos. Métodos: Esta es una revisión integradora cuyo tema fue investigado en la base de datos de PubMed y Scielo, con descriptores "Calidad de vida Y Diabetes Mellitus", solo se incluyeron artículos de los últimos tres años. Resultados: Los artículos desarrollados a través de cuestionarios mostraron que la calidad de vida en los diabéticos es menor que en los pacientes no diabéticos, principalmente debido al cambio en el estilo de vida que genera la enfermedad. Este hecho también está relacionado con las complicaciones presentes en el tratamiento incorrecto de la enfermedad, así como con la terapia farmacológica en sí, con los cambios en el estilo de vida que se imponen y con la ansiedad

1 Universidade Tiradentes (UNIT), Aracaju - SE. *E-mail: luumaribeiro@gmail.com

SUBMETIDO EM: 6/2020

ACEITO EM: 6/2020

PUBLICADO EM: 8/2020 
presente en la mayoría de estos pacientes. Por lo tanto, tales individuos tienen que lidiar con las complicaciones físicas y mentales que determina la enfermedad, causando daños a la forma de vida. Consideraciones Finales: los pacientes diabéticos tienen una calidad de vida más baja, tanto física como emocionalmente, en comparación con las personas que no tienen la enfermedad.

Palabras clave: Calidad de vida, Diabetes mellitus, Complicaciones.

\section{INTRODUÇÃO}

Diabetes Mellitus (DM) é uma doença metabólica caracterizada por hiperglicemia persistente, resultante da resistência de insulina e/ou de defeitos na secreção desta (GROSS J, et al., 2002). A desregulação crônica da insulina está associada a algumas complicações microvasculares e macrovasculares, como retinopatia, nefropatia, neuropatia, doença coronariana, doença cerebrovascular e doença arterial periférica (CORREA K, et al., 2017; SOCIEDADE BRASILEIRA DE DIABETES, 2017).

Segundo a literatura, é a doença crônica mais comum mundialmente - um em cada dez adultos são diabéticos - e é responsável por 3,5\% das mortes. Ademais, é considerado um importante e crescente problema de saúde para todos os países, e cerca de $75 \%$ dos casos são de países em desenvolvimento, nos quais deverá ocorrer o maior aumento dos casos de diabetes nas próximas décadas. O aumento da prevalência do DM deve-se principalmente à maior expectativa dos indivíduos, além de hábitos de vida da sociedade atual, como aumento da obesidade, maior carga horária no trabalho, gerando, assim, altas taxas de sedentarismo e menor prática de uma alimentação saudável (ABEDINI MR, et al., 2020; SOCIEDADE BRASILEIRA DE DIABETES, 2017).

O conceito de qualidade de vida $(\mathrm{QV})$ tem sido foco de diversos estudos nos últimos tempos, e vem recebendo definições diferentes a depender da época e do contexto onde tem sido utilizado. A conceituação mais aceita atualmente é QV como percepção que o indivíduo tem em sua posição na vida, no contexto e cultura nos quais vive, com relação a seus objetivos, expectativas e preocupações (LIMA LR, et al., 2018).

A avaliação da QV é considerada medida importante para estimar o resultado do bem estar do paciente, além de identificar deficiências específicas do mesmo ao longo da evolução de sua doença. Sabe-se que na prática médica atual, devido às restrições de tempo durante as consultas, a avaliação da QV parece bastante improvável ou difícil de ser executada, e os médicos frequentemente a inferem como parte do processo de tomada de decisão, sem perguntar diretamente aos pacientes (ABEDINI MR, et al., 2020; TAMIR O, et al., 2018).

Sabe-se que tanto o diabetes tipo 1 quanto o tipo 2 ocasionam redução da qualidade de vida do indivíduo quando comparado com pessoas que não possuem a doença. Tal fato está relacionado principalmente às complicações presentes na inadequação do tratamento, assim como à própria terapia farmacológica, às alterações de estilo de vida que são impostas e à ansiedade presente em grande parte desses pacientes (MASCARENHAS LPG, et al., 2018; MENEZES M, et al., 2019).

Outra razão do maior comprometimento da QV é que o DM está frequentemente associado a limitações, estresse social e financeiro, desconforto emocional e outras alterações psicológicas. Tais alterações, por conseguinte, estão diretamente ligadas à menor aceitação da doença, logo, a um menor grau de receptividade ao tratamento (MASCARENHAS LPG, et al., 2018; SANTOS RLB, et al., 2017).

Logo, a QV é um desafio diário do indivíduo diabético, pois nem sempre o diagnóstico e enfrentamento da doença são de fácil adaptação. Avaliá-la, portanto, é um meio de cuidado do paciente como um todo, de modo que haja identificação do manejo da doença crônica, além da monitorização precoce do sofrimento físico e emocional que a doença possa estar causando naquele momento (ABEDINI MR, et al., 2020; TONETTO IFA, et al., 2019).

Desse modo, diante da elevada prevalência mundial do diabetes do mundo, assim como do impacto das mudanças de hábitos e das complicações de vida destes indivíduos, propõe-se um estudo para avaliar a 
qualidade de vida dessa população, bem como identificar as principais causas que possam relacionar-se ao seu comprometimento.

\section{MÉTODOS}

Trata-se de uma revisão literária com coleta de informações a partir do levantamento bibliográfico e seguindo os moldes de uma revisão integrativa, método pelo qual se analisa um tema estudado em pesquisas anteriores, com o objetivo de compreender mais determinado assunto.

A revisão foi aprofundada a partir dos seguintes passos: identificação do tema e seleção da questão de pesquisa; estabelecimento de critérios de inclusão e exclusão; identificação e seleção dos estudos; categorização dos estudos selecionados; análise e interpretação dos resultados e apresentação da revisão/síntese do conhecimento.

A pesquisa foi feita de acordo com a base de dados Scientific Eletronic Library Online (SCIELO) e National Library of Medicine (PUBMED), com descritores "Qualidade de vida AND Diabetes Mellitus" e "Quality of life AND Diabetes Mellitus", respectivamente.

Foram incluídos apenas os artigos publicados entre janeiro de 2017 e janeiro de 2020, por se tratar de um período com dados atualizados. Ademais, outro critério foi possuir como tema principal a avaliação da qualidade de vida em diabéticos (tipos 1 e 2) e utilizar questionários validados para mensuração desta. Critérios de exclusão foram os artigos publicados antes de 2017 e aqueles que não tiveram como tema principal a qualidade de vida sendo mensurada através de questionários ou apresentaram associação da qualidade de vida com alguma complicação específica do diabetes.

Inicialmente, a busca de artigos que se adequassem aos critérios de inclusão se deu no PUBMED. Os filtros utilizados foram: texto completo gratuito, últimos cinco anos, humanos e em adultos maiores que 19 anos. A procura resultou em 978 artigos, dos quais apenas 37 apresentavam como tema principal a qualidade de vida de diabéticos determinada por questionários. Destes, ainda foram excluídos nove, por terem sido escritos antes de 2017. Restaram, então, 28 artigos aptos para o estudo. Em seguida, a pesquisa foi feita no SCIELO com os seguintes filtros: idiomas português e inglês, publicados nos últimos três anos. Como resultados, foram obtidos 39 artigos, dos quais apenas oito se adequaram nos critérios de inclusão. Destes, quatro foram excluídos por serem repetidos, sobrando quatro artigos para serem avaliados nesta plataforma. Vale destacar, então, que foram separados um total de 32 artigos para o estudo em questão.

\section{RESULTADOS E DISCUSSÃO}

A qualidade de vida agrega a percepção subjetiva do indivíduo do bem-estar físico, emocional e social, e inclui um componente cognitivo e emocional que está se tornando cada vez mais importante para os cuidados de saúde para pacientes diabéticos (SANTOS RLB, et al., 2017). A maioria das condições que interferem na QV são, em geral, modificáveis.

Segundo Stojanovic M, et al. (2018) os principais fatores que podem contribuir para uma menor qualidade de vida na população diabética são sexo feminino, menor status econômico, menor nível de educação, falta de condição física associada à ausência de exercício físico e complicações diabéticas macro ou microvasculares.

Estudos afirmam que diabéticos possuem menor QV quando comparados a indivíduos que não possuem a doença e a pré-diabéticos (Figura 1). Pesquisa feita por Bak E, et al. (2019) evidenciou que mais de $80 \%$ dos diabéticos entrevistados relataram que sua QV melhoraria caso não tivessem diabetes. Isso pode ser explicado pela dificuldade que tal indivíduo tem de lidar com o diagnóstico e tratamento ao longo do tempo, além de possuir outras comorbidades associadas que podem diminuir seu bem estar. O DM é uma doença crônica severa tanto pelo comprometimento físico ou emocional, quanto em relação ao autocuidado do indivíduo (MAKRILAKIS K, et al., 2018; PRASANNAKUMAR HR, et al., 2017). 
Figura 1 - Comparação do score do questionário The 15D Instrument of Health-Related Quality of Life (HRQOL-15D) entre pacientes diabéticos, pré diabéticos e não diabéticos.

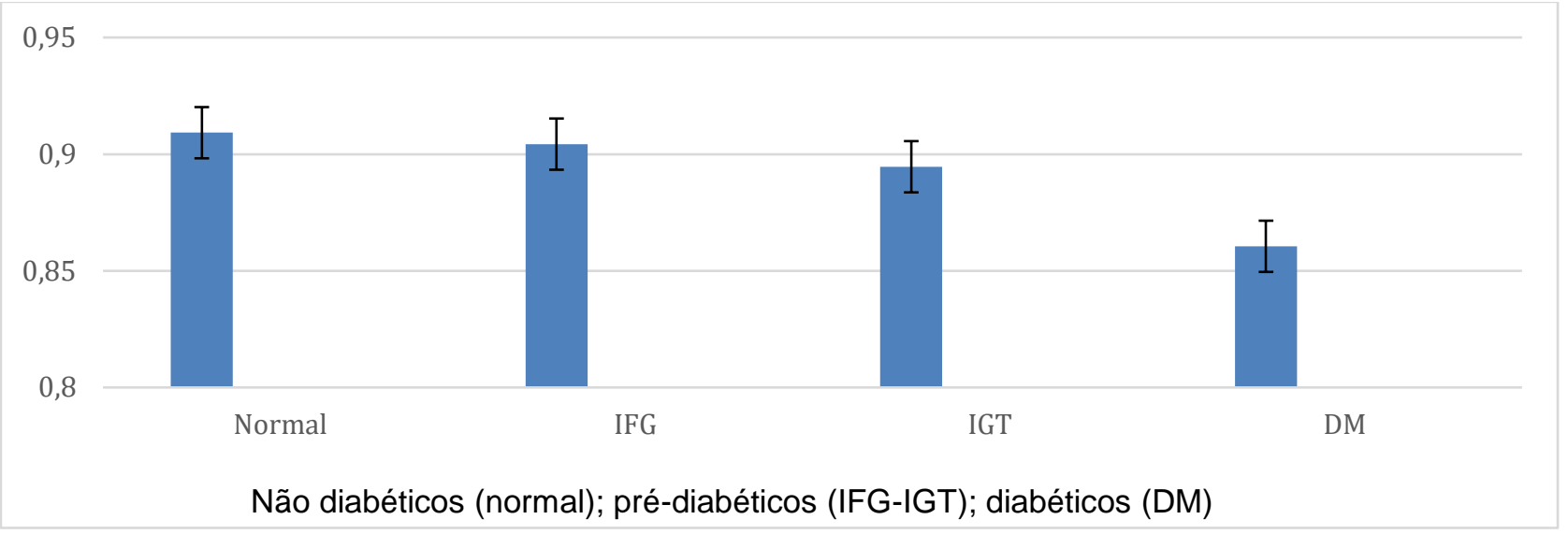

Fonte: Ribeiro LMA, et al., 2020. Baseado em Makrilakis K, et al., 2018 e BMC Endocrine Disorders.

Quanto ao tempo de diagnóstico, estudos mais recentes sugerem que um maior tempo de doença, em geral maior que dez anos, está relacionado à uma diminuição da QV. Tal fato pode estar associado à maior presença de complicações, má adesão ao tratamento, com consequente pior controle metabólico da glicemia, assim como aos próprios efeitos colaterais dos medicamentos a longo prazo (ABEDINI MR, et al., 2020; GEBREMEDHIN T, et al., 2019; LIMA LM, et al., 2018).

A associação da QV com a idade dos diabéticos é um tema bastante controverso na literatura. Moreschi C, et al. (2018) sugerem que, com o aumento da idade, em geral, há diminuição significativa da qualidade de vida, provavelmente relacionada ao maior tempo de diagnóstico e às dificuldades de adesão terapêutica, como a de fazer atividades físicas, associado à dificuldade em aceitar mudanças que há muito faziam parte de uma rotina consolidada. $O$ aumento da idade traz consigo características peculiares da redução do sistema fisiológico, como fraqueza física, dores crônicas e dependência do indivíduo, exigindo apoio de um cuidador, o que culmina em uma percepção alterada de si próprio enxergando-se incapaz de cumprir com seus propósitos (LIMA LM, et al., 2018; ZHUANG Y, et al., 2020).

Já Pimienta EG, et al. (2019) acreditam que pacientes mais jovens apresentam pior QV por serem incapazes de seguir o tratamento à risca, devido aos conflitos inerentes à idade, ao menor tempo de aceitação de seu diagnóstico, assim como à dificuldade de adequação de mudanças de hábitos para a terapêutica eficaz. Do contrário, pacientes mais velhos teriam mais oportunidade de processar e se adaptar à sua condição clínica. Quando o diabetes é diagnosticado em adolescentes, por exemplo, gera um impacto psicossocial, uma vez que desencadeia modificações em várias esferas da identidade pessoal. Sendo assim, não se pode concluir que a idade seja fator de risco independente para redução da $Q V$, e deve ser avaliada de acordo com o contexto em que se apresenta (MENEZES M, et al., 2019; SOUZA MA, et al., 2019).

Por outro lado, a relação entre presença de complicações do DM e redução de QV é bastante prevalente na literatura. O Diabetes Mellitus é uma doença crônica que pode levar a graves complicações, tanto físicas como mentais (MAKRILAKIS KAKF, et al., 2018). De acordo com Pimienta EG, et al. (2019) as complicações mais encontradas nessa população foram neuropatia, como comorbidade física, e depressão, como psiquiátrica. A maioria dos estudos refere que diabéticos que possuem complicações ou outras comorbidades associadas têm diminuição do bem estar. Tal efeito na QV é pior quanto maior o número de complicações.

Estudo feito por Jiao F, et al. (2017) demonstrou que indivíduos com alterações macrovasculares, como doença cardíaca e acidente vascular cerebral, apresentaram menor score de QV, ao passo que aqueles com comprometimento microvascular, como nefropatia e retinopatia, somente apresentaram redução na QV quando o nível da doença era grave e irreversível. Isso reflete o nível de sintomatologia relacionada às complicações, visto que aquelas que se apresentam assintomáticas não ocasionam diminuição da QV por não interferirem muito na diminuição da autonomia e da participação social (DHILLON H, et al., 2019). 
Portanto, a presença de comorbidades associa-se à menor QV devido a seu impacto na mobilidade, autonomia, participação social e acúmulo de remédios para tratamento de diversas doenças concomitantes (ALSHAYBAN D, et al., 2020; LIMA LM, et al., 2018; THAPA S, et al., 2019).

Associado ao número de complicações está o número de hospitalizações devido a complicações do DM. A literatura relata que pacientes com histórico de hospitalizações possui QV mais baixa. Uma possível explicação é que a internação pode ser indicativa de mau controle glicêmico. Além do mais, durante a internação, o indivíduo é de certa forma, privado de seu convívio social, o que leva à sensação de impotência e sentimento de solidão (ABEDINI MR, et al., 2020; SOUZA MA, et al., 2019).

Outro fator que confere diminuição da QV em pacientes com DM seria o gênero feminino. Isso é justificado pela maior prevalência de diabetes nessa população, seja por estarem mais presentes nas Unidades Básicas de Saúde, o que favorece seu diagnóstico, ou porque são mais propensas a apresentarem transtornos psicológicos, como ansiedade e depressão (SANTOS RLB, et al., 2017; ARIFIN B, et al., 2019).

Correa K, et al. (2017) também associam ao fato de que, em geral, as mulheres têm maior sobrecarga com o cuidado à família e, por isso, deixariam de ter o autocuidado a respeito das próprias doenças. Alguns estudos ainda afirmam que a menor QV está atrelada ao menor tempo de cuidado da mulher, visto que elas trabalham e fazem trabalhos domésticos, e assim, não teriam tempo para prática de atividades físicas (ALSHAYBAN D, et al., 2020; LU Y, et al., 2017).

No que diz respeito ao tratamento, sabe-se que um bom controle glicêmico está associado a melhores escores de QV. Tonetto IFA, et al. (2019) evidenciaram que para maiores taxas de hemoglobina glicada havia maior percepção do comprometimento e da gravidade da doença. Valores de hemoglobina glicada dentro da normalidade (abaixo de sete porcento), assim como a prática de atividade física de no mínimo três vezes por 30 minutos durante a semana, associada a um índice de massa corporal (IMC) abaixo de $18,4 \mathrm{~kg} / \mathrm{m}^{2} \mathrm{causam}$ um aumento na QV do indivíduo; ao passo que sedentarismo, IMC na faixa normal ou acima e elevados índices de hemoglobina glicada são fatores de risco para uma redução na QV do paciente (ANDERSON BJ, et al., 2017; CORREA K, et al., 2017; SOUZA MA, et al., 2019) .

De acordo com estudo desenvolvido por Gebremedhin T, et al. (2019), o IMC está relacionado a uma menor QV pela menor mobilidade e pela insatisfação com a aparência do próprio corpo. É importante lembrar que a maioria dos diabéticos tipo 2 apresentam sobrepeso ou obesidade, o que reflete um IMC acima da média, além de risco cardiovascular alto, o que gera maior risco de complicações, e, portanto, apresentam um risco maior de diminuição acentuada da QV.

Quanto aos medicamentos em uso, a aplicação de insulina como monoterapia ou associada a hipoglicemiantes orais apresentou redução na QV de diabéticos (KHUNKAEW S, et al., 2019; TONETTO IFA, et al., 2019). A possível explicação deve-se ao fato de que a insulina é muito utilizada pelo melhor controle glicêmico, entretanto, apresenta desvantagem, como certa complexidade na aplicação e dor (DHILLON H, et al., 2019). Além disso, Alshayban $D$, et al. (2020) referiram que os pacientes também apresentavam medo de alteração de peso e de eventos hipoglicemiantes com o uso da injeção.

Ademais, alguns autores referem associação da diminuição da qualidade de vida com menor nível de classe econômica e/ou escolaridade. Tal pensamento está atrelado à maior proporção de sedentarismo nessa classe, assim como ao déficit de conhecimento a respeito da doença, o que acarreta má adesão ao tratamento (SANTOS, RLB, et al., 2017).

Stojanovic M, et al. (2018) ainda explicam essa interação por classes inferiores terem acesso inferior aos serviços de saúde e inadequação aos planos de tratamento. De outro modo, diabéticos com classe social mais alta e/ou escolaridade com ensino superior têm uma compreensão melhor do seu diagnóstico, logo, uma atitude mais consciente em relação à terapia adotada, além de possuírem mais fácil acesso a serviços de saúde, o que facilita o acompanhamento da doença por meio de exames regulares e cobre o alto custo de alguns tratamentos (ARIFIN B, et al., 2019; ABEDINI MR, et al., 2020; IQBAL Q, et al., 2017). 
Outro assunto abordado na literatura é ligação da qualidade de vida com o suporte familiar. Acredita-se que indivíduos que possuem um bom suporte familiar para o enfrentamento da doença têm melhor QV. Anderson BJ, et al. (2017) relataram que a pior QV estava relacionada com existência de conflitos familiares. As contribuições familiares e apoio emocional têm sido apontadas como fatores de melhora do controle metabólico dos pacientes, tal fato poderia estar relacionado a melhores escores de QV em diabéticos tipo 1 quando comparados ao tipo 2, pois naqueles, devido a uma idade de diagnóstico mais precoce, haveria um maior apoio familiar ao diagnóstico da doença (MENEZES M, et al., 2019; MURILLO M, et al., 2017).

Além disso, Santos RLB, et al. (2017) afirmaram que o estado civil interfere na adesão ao tratamento e na dinâmica familiar, enquanto o psicossocial influencia nas variações glicêmicas, sendo o ambiente familiar um estímulo para o autocuidado.

Em geral, os médicos dedicam-se a prevenir as complicações secundárias da doença, de modo que pedem constantemente parâmetros bioquímicos para monitoramento da glicemia. Porém, de forma paradoxal, esses mesmos médicos deixam para segundo plano a monitorização da qualidade de vida do paciente, tendendo a sub ou superestimá-la por julgamentos próprios. Tal percepção médica da QV dos pacientes foi avaliada através de uma pesquisa feita por Tamir O, et al. (2018), que evidenciou melhor percepção por parte dos especialistas em diabetes quando comparados com os médicos da atenção básica. Isso pode refletir um melhor treinamento desse grupo de profissionais por possuírem um conhecimento íntimo da doença em questão.

Entretanto, quando se avaliam indivíduos acompanhados em serviços especializados, observa-se menor QV destes em relação àqueles tratados na atenção primária. Isso pode ser explicado pela maior prevalência de complicações e de doença mais grave ou de difícil tratamento naqueles que são tratados no serviço especializado (ARIFIN B, et al., 2019).

Por fim, a literatura mostra grande associação do DM com alterações psicológicas, sendo estas tanto causas como consequências da pior QV. Existem algumas explicações sobre o mecanismo biológico de sofrimento emocional em diabéticos tipo 2. Uma teoria bem fundamentada envolve níveis aumentados de hormônio adrenocorticotrópico (ACTH), promovendo a atividade do eixo hipotálamo-hipófise-adrenal (HPA), e o próprio eixo modifica os níveis de glicose, realimentando o círculo vicioso. Outra possível explicação é o nível aumentado de fator de necrose tumoral alfa (TNFa), devido ao caráter inflamatório crônico da doença. O TNFa, por usa vez, estimula a interrupção da barreira hematoecefálica, o que pode acarretar a perda de regulação do transporte de outros sinais inflamatórios na BBB e agravar a carga alostática no eixo HPA, o que levaria à sua desregulação (PIMIENTA EG, et al., 2019).

Segundo Zurita-Cruz JN, et al. (2018) o diagnóstico de Diabetes Mellitus favorece o aparecimento de problemas emocionais, principalmente a depressão. Portanto, transtornos psiquiátricos, principalmente depressão, ansiedade e estresse podem ocasionar em pior adesão ao tratamento, mau controle glicêmico e aumento de custos com saúde. Assim como o próprio tratamento restritivo, doses diárias de insulina, medo de episódios hipoglicêmicos e hiperglicemias frequentes podem exacerbar a morbidade psiquiátrica existente.

Ademais, Thapa S, et al. (2019) relataram que ter histórico familiar de diabetes estava associado a um pior estado mental dos pacientes, pois estes se preocupavam com as condições que impactavam a qualidade de vida dos familiares e deles mesmos. O impacto de assumir o diagnóstico e o tratamento causa inúmeras respostas emocionais no paciente, podendo levar à baixa autoestima, falta de exaustão e motivação emocional, dificultando o processo de adaptação à doença e ao tratamento (PIMIENTA EG, et al., 2019).

\section{CONSIDERAÇÕES FINAIS}

Diante do exposto, foi possível perceber que há de fato uma redução da qualidade de vida de pacientes diabéticos. Dentre os principais fatores de piora da qualidade de vida estão o gênero feminino, tempo maior de diagnóstico, maior número de complicações, mau controle glicêmico, escolaridade mais baixa, pouco suporte familiar e associação com transtornos psiquiátricos. Desse modo, é de extrema importância avaliar a 
QV desses indivíduos, de modo que haja identificação precoce de fatores que interfiram nesta, e assim, possam ser instituídas estratégias na rotina da equipe quanto ao cuidado com o paciente, de modo que a melhoria da QV estará atrelada à maior satisfação do diabético, bem como ao comprometimento da mesma ao autocuidado.

\section{REFERÊNCIAS}

1. ABEDINI MR, et al. The quality of life of the patients with diabetes type 2 using EQ-5D-5L in Birjand. Health Qual Life Outcomes. 2020; 18 (1): 18.

2. ALSHAYBAN D, et al. Health-related quality of life among patients with type 2 diabetes mellitus in Eastern Province, Saudi Arabia: A cross-sectional study. PLoS One. 2020; 15 (1): e0227573.

3. ANDERSON BJ, et al. Factors Associated With Diabetes-Specific Health-Related Quality of Life in Youth With Type 1 Diabetes: The Global TEENs Study [published correction appears in Diabetes Care. 2018 Jan 10;]. Diabetes Care. 2017; 40(8): 1002-1009.

4. ARIFIN B, et al. Health-related quality of life in Indonesian type 2 diabetes mellitus outpatients measured with the Bahasa version of EQ-5D. Qual Life Res. 2019; 28(5): 1179-1190.

5. BĄK E, et al. An assessment of diabetes-dependent quality of life (ADDQoL) in women and men in Poland with type 1 and type 2 diabetes. Ann Agric Environ Med. 2019; 26(3): 429-438.

6. CORREA K, et al. Qualidade de vida e características dos pacientes diabéticos. Ciência \& Saúde Coletiva, [s.I.], 2017; 22(3): 921-930. Fap UNIFESP (SciELO).

7. DHILLON H, et al. Quality of Life and Associated Factors among Primary Care Asian Patients with Type 2 Diabetes Mellitus. Int J Environ Res Public Health. 2019; 16 (19): 3561.

8. GEBREMEDHIN T, et al. Health-related quality of life and its associated factors among adult patients with type II diabetes attending Mizan Tepi University Teaching Hospital, Southwest Ethiopia. BMJ Open Diabetes Res Care. 2019; 7 (1): e000577.

9. GÓMEZ-PIMIENTA E, et al. Decreased Quality of Life in Individuals with Type 2 Diabetes Mellitus Is Associated with Emotional Distress. International Journal of Environmental Research and Public Health, [s.I.], 2019; 16(15): 26522660.

10. GROSS JL, et al. Diabetes Melito: Diagnóstico, Classificação e Avaliação do Controle Glicêmico. Arq Bras Endocrinol Metab, São Paulo, 2002; 46(1): 16-26.

11. HUBER MB, et al. Health-Related Quality of Life of the General German Population in 2015: Results from the EQ-5D5L. Int J Environ Res Public Health. 2017; 14(4): 426.

12. IQBAL $Q$, et al. Profile and predictors of health related quality of life among type II diabetes mellitus patients in Quetta city, Pakistan. Health Qual Life Outcomes. 2017; 15(1): 142.

13. JIAO F, et al. Health-related quality of life and health preference of Chinese patients with diabetes mellitus managed in primary care and secondary care setting: decrements associated with individual complication and number of complications. Health Qual Life Outcomes. 2017; 15(1): 125.

14. KHUNKAEW S, et al. Demographic and clinical predictors of health-related quality of life among people with type 2 diabetes mellitus living in northern Thailand: A cross-sectional study. Health Qual Life Outcomes. $2019 ; 17$ (1): 177.

15. LIMA LR, et al. Qualidade de vida e tempo desde o diagnóstico de Diabetes Mellitus em idosos. Rev. bras. geriatr. gerontol., Rio de Janeiro, 2018; 21(2): 176-185.

16. LU Y, et al. Health-related quality of life in type-2 diabetes patients: a cross-sectional study in East China. BMC Endocrine Disorders. 2017; 17 (1): 38.

17. MASCARENHAS LPG, et al. Health-related quality of life in a cohort of youths with type 1 diabetes. Rev. Assoc. Med. Bras., São Paulo, 2018; 64(11): 1038-1044.

18. MAKRILAKIS, K., et al. Comparison of health-related quality of Life (HRQOL) kamong patients with pre-diabetes, diabetes and normal glucose tolerance, using the 15D-HRQOL questionnaire in Greece: the DEPLAN study. BMC Endocr Disord 18, 32 (2018).

19. MENEZES M, et al. Qualidade de vida e diabetes mellitus: autopercepção de adolescentes de uma cidade do sul do Brasil. Psic.: Teor. e Pesq., Brasília, 2019; 35: e35430.

20. MORESCHI C, et al. Family Health Strategies: Profile/quality of life of people with diabetes. Rev Bras Enferm. 2018; $71(6): 2899-2906$.

21. MURILLO M, et al. Health-related quality of life (HRQOL) and its associated factors in children with Type 1 Diabetes Mellitus (T1DM). BMC Pediatr. 2017; 17(1): 16.

22. PRASANNAKUMAR HR, et al. Patient Self-reported quality of life assessment in Type 2 diabetes mellitus: A pilot study. Niger J Clin Pract. 2018; $21(3)$ : 343-349.

23. PAULA JS, et al. Correlation between parameters of self-monitoring of blood glucose and the perception of healthrelated quality of life in patients with type 1 diabetes mellitus. Arch Endocrinol Metab. 2017; 61(4): 343-347.

24. RAYMAKERS AJN, et al. Factors influencing health-related quality of life in patients with Type 1 diabetes. Health Qual Life Outcomes. 2018; 16(1): 27.

25. REIS ACD, et al. Comparison of quality of life and functionality in type 2 diabetics with and without insulin. Rev Assoc Med Bras (1992). 2019; 65 (12): 1464-1469. 
26. SANTOS RLB, et al. Fatores associados à qualidade de vida de brasileiros e de diabéticos: evidências de um inquérito de base populacional. Ciência \& Saúde Coletiva, [s.I.], 2017; 24(3): 1007-1020. Fap UNIFESP (SciELO).

27. SOCIEDADE BRASILEIRA DE DIABETES-SBD. Diretrizes da Sociedade Brasileira de Diabetes: 2017-2018. In: Milech A, Oliveira JEP, Vencio S, (Org.). São Paulo: AC Farmacêutica.

28. SOUZA MA, et al. Health-related quality of life of adolescents with type 1 diabetes mellitus. Rev. Latino-Am. Enfermagem, Ribeirão Preto, 2019; 27: e3210.

29. STOJANOVIĆ M, et al. Impact of socio-demographic characteristics and long-term complications on quality of life in patients with diabetes mellitus. Cent Eur J Public Health. 2018; 26(2): 104-110.

30. SVEDBO ENGSTRÖM M, et al. Health-related quality of life and glycaemic control among adults with type 1 and type 2 diabetes - a nationwide cross-sectional study. Health Qual Life Outcomes. 2019; 17(1): 141.

31. THAPA S, et al. Health-related quality of life among people living with type 2 diabetes: a community based crosssectional study in rural Nepal. BMC Public Health. 2019; 19(1): 1171.

32. TONETTO IFA, et al. Quality of life of people with diabetes mellitus. Rev. esc. enferm. USP, São Paulo, 2019; 53: e03424.

33. VIGEN CLP, et al. Psychosocial and Behavioral Correlates of A1C and Quality of Life Among Young Adults With Diabetes. Diabetes Educ. 2018; 44(6): 489-500.

34. ZHUANG Y, et al. Health-related quality of life in older Chinese patients with diabetes. PLoS One. 2020; 15(2): e0229652.

35. ZURITA-CRUZ JN, et al. Saúde e resultados de qualidade de vida comprometimento da qualidade de vida no diabetes mellitus tipo 2: um estudo seccional. Resultados de vida com qualidade de saúde. 2018; 16(1): 94. 